\title{
From waves to avalanches: two different mechanisms of sandpile dynamics
}

\author{
Mario De Menech ${ }^{1}$ and Attilio L. Stella ${ }^{1,2}$ \\ ${ }^{1}$ INFM-Dipartimento di Fisica, \\ Università di Padova, I-35131 Padova, Italy \\ 2 The Abdus Salam ICTP, P. O. Box 563, I-34100, Trieste, Italy, \\ Sezione INFN, Università di Padova, I-35131 Padova, Italy
}

(October 25, 2018)

Time series resulting from wave decomposition show the existence of different correlation patterns for avalanche dynamics. For the $d=2$ Bak-Tang-Wiesenfeld model, long range correlations determine a modification of the wave size distribution under coarse graining in time, and multifractal scaling for avalanches. In the Manna model, the distribution of avalanches coincides with that of waves, which are uncorrelated and obey finite size scaling, a result expected also for the $d=3$ Bak et al. model.

PACS numbers:05.65.+b,05.40.-a,45.70.Ht,64.60.Ak

Full information on a self-organized critical (SOC) process [1] is contained in the time series, if the time step is the most microscopic conceivable. The self-similarity of such a process, due to its intermittent, avalanche character, should be revealed by the scaling of the time autocorrelation or its power spectrum. In spite of this, time series analyses have been seldom performed on sandpiles or similar systems, and mostly concerned the response to finite, random external disturbances, i.e. the problem of $1 / f$ noise [2]. Most efforts in the characterization of SOC scaling concentrated on probability distribution functions (PDF's) of global properties of the avalanche events, which occupy large intervals of the microscopic evolution time [3]. The numerical analysis of such PDF's is often difficult, and universality issues can not be easily solved. The situation is even more problematic if, like for the two-dimensional (2D) Bak-Tang-Wiesenfeld sandpile (BTW) [1], the usually assumed finite size scaling (FSS) form reveals inadequate for the PDF's, and needs to be replaced by a multifractal one [4]. These findings rise the additional issue of why the 2D BTW displays such multifractal scaling, while apparently very similar sandpiles, like the Manna model (M) [5], do not 国6,

In recent theoretical approaches to the $\mathrm{BTW}$ and other Abelian sandpiles [8], a major role has been played by the waves of toppling into which avalanches can be decomposed [9]. For the BTW, the PDF's of waves, as sampled from a large collection of successive avalanches, obey FSS with exactly known exponents [10]. By analyzing the succession of waves as a stationary time series, one could hope to determine the statistical properties of avalanches. However, so far, no precise information on the correlations of such series was obtained.

In this Letter we generalize the wave description to the $\mathrm{M}$ in $2 \mathrm{D}$. The study of the respective wave time series reveals that BTW and $\mathrm{M}$ are prototypes of two very different scenarios for avalanche dynamics. In the $\mathrm{M}$ case, successive wave sizes are totally uncorrelated. As a consequence, avalanche and wave PDF's have identical scaling properties, consistent with FSS. For the BTW, to the contrary, wave sizes show long range correlations and persistency in time. PDF's of "block variables" given by sums of $n$ successive wave sizes show that these correlations are responsible for the fact that avalanche scaling differs from the wave one and has multifractal features. For the 3D BTW, on the other hand, our results suggest validity of a scenario identical to the $\mathrm{M}$ one, and lead to conjecture exact avalanche exponents coinciding with those of the wave PDF.

The 2D BTW [1] is defined on a square $L \times L$ lattice. An integer $z_{i}$, the number of grains, is assigned to site $i$. Starting from a stable configuration $\left(z_{i} \leq z_{c}=4\right.$, $\forall i)$, a grain is added at a randomly chosen site; after each addition, all sites exceeding the stability threshold, $z_{k}>z_{c}$, undergo toppling, distributing one grain to each one of the nearest neighbors. The topplings, which dissipate grains when occurring at the edges, continue until all sites are stable, and a new grain is added. The $s$ topplings between two consecutive additions form an avalanche. After many additions, the system organizes into a stationary critical state.

Manna [5] studied a two-state version, M, of the sandpile. The sites can be either empty or occupied; grains are added randomly, and when one of them drops onto an occupied site, a "hard core" repulsion pushes two particles out to randomly chosen nearest neighbors. Compared to the BTW, in which toppling is deterministic, this model has an extra stochastic ingredient in the microscopic evolution, and $z_{c}=1$.

One can define a special ordering in the topplings of the BTW by introducing the wave decomposition of avalanches [9]. After the site of addition, $O$, topples, any other unstable site is allowed to topple except possibly $O$. This is the first wave of toppling. If $O$ is still unstable, it is allowed to topple once again, leading to the second wave. This continues until $O$ becomes stable. Thus, an avalanche is broken into a sequence of waves. During a wave, sites topple only once, and for an $m$-wave avalanche $s=\sum_{k=1}^{m} s_{k}$, where $s_{k}$ are the topplings of the $k$-th wave. Following the definitions for the BTW, we implement here a wave decomposition of avalanches also for the M. Unlike for the BTW, sites involved in 
a wave may topple more than once. Furthermore, the toppling order chosen implies now a peculiar sequence of stable configurations visited upon addition of grains, but the realization probabilities of possible configurations are independent of this order [8].

The BTW wave size PDF has FSS form $P_{w}(s) \sim$ $s^{-\tau_{w}} p_{w}\left(s / L^{D_{w}}\right)$, with $\tau_{w}=1, D_{w}=2$ in $2 \mathrm{D}$, and $p_{w}$ a suitable scaling function [9, 10]. For the $\mathrm{M}$ waves we also found a PDF obeying FSS, with $\tau_{w}=1.31 \pm 0.02$ and $D_{w}=2.75 \pm 0.01$. These exponents are remarkably consistent with those estimated for avalanches [5, 6]. Such a coincidence certainly does not apply to the BTW. Attempts to derive exact BTW avalanche exponents within FSS were based on the observation that often waves within an avalanche show a final, long contraction phase, and on a scaling assumption for the corresponding $s_{k}-s_{k+1}$ 11. A more adequate ansatz for the conditional PDF of $s_{k+1}$ given $s_{k}$ [12, and Markovianity assumptions, did not help in better characterizing avalanche scaling along these lines 13 . In fact the $2 \mathrm{D}$ BTW obeys a multifractal form of scaling, which is not catched by such simplified schemes [4].

The wave time series $\left\{s_{k}\right\}$ provide coarse grained dynamical descriptions. In the $L \rightarrow \infty$ limit, these descriptions are infinitely rescaled with respect to those at microscopic time scale, but still infinitely finer than the mere records of successive avalanche sizes. This intermediate time scale reveals essential in order to understand the dynamics indside avalanches, whose size sequence we found to be uncorrelated in the sense discussed below for waves. The microscopic scale gave no significant results in comparing the two models, since, at that level, similar strong correlations exists in both of them, due to the parallel updating algorithm.

We determined for BTW and M the autocorrelation

$$
C(t, L)=\frac{\left\langle s_{k+t} s_{k}\right\rangle_{L}-\mu^{2}}{\left\langle s_{k}^{2}\right\rangle_{L}-\mu^{2}}
$$

with $t=1,2, \ldots$, and $\mu=\left\langle s_{k}\right\rangle_{L}$, the time averages being taken over up to $10^{7}$ waves for $L=128,256,512,1024$ and 2048. A first, striking result is that, as soon as $t>0$ $(C(0, L)=1$ by normalization), waves are uncorrelated in the M. Indeed, as $L$ grows, $C$ manifestly approaches 0 as soon as $t>0$ (Fig. 1). To the contrary, $C$ is long range for the BTW, because it approaches 0 only for $t$ exceeding the maximum number of waves in an avalanche (Fig. 1), which we found to scale $\sim L$, for $L \rightarrow \infty$. For the BTW we further tested the FSS form

$$
C(t, L)=t^{-\tau_{c}} g\left(t / L^{D_{c}}\right) \quad(t, L \gg 1),
$$

on the basis of the $L \rightarrow \infty$ scaling of the moments 14,4

$$
\left\langle t^{q}\right\rangle_{L}=\sum_{t} C(t, L) t^{q} \sim L^{\sigma_{c}(q)} .
$$

FSS would imply the piecewise linear form

$$
\sigma_{c}(q)= \begin{cases}D_{c}\left(q-\tau_{c}+1\right) & \text { if } q \geq \tau_{c}-1 \\ 0 & \text { if } q<\tau_{c}-1\end{cases}
$$

Fig. 2 shows the extrapolated $\sigma_{c}(q)$, which has an approximately linear part for $1 \leq q \leq 4$, consistent with $D_{c}=1.02 \pm 0.05$ and $\tau_{c}=0.40 \pm 0.05$. The curvature for $q<1$ is due to the fact that, for finite $L$, a logarithm $\left(\tau_{c}=0\right)$ can not be easily distinguished from a power law with $\tau_{c} \gtrsim 0$. The inset of Fig. 2 shows this logarithmic growth of $\left\langle t^{q}\right\rangle_{L}$. We conclude that, for the BTW, a simple power law tail $C(t, \infty) \sim t^{-\tau_{c}}$, is a first, rough, approximation. The increment $y(t)=\sum_{k=1}^{t} s_{k}$ is comparable to the trail of a fractional Brownian motion with Hurst exponent $H=\left(2-\tau_{c}\right) / 2$ [15], such that $H=0.80 \pm 0.03$ for the BTW. $H$ can be measured directly from the fluctuation

$$
F(t, L)=\left[\left\langle\Delta y(t)^{2}\right\rangle_{L}-\langle\Delta y(t)\rangle_{L}^{2}\right]^{1 / 2},
$$

with $\Delta y(t)=y(k+t)-y(k)$ [15], which should scale as $F(t, \infty) \sim t^{H}$. Fig. 3 reports tests of this scaling for $L=2048$. For the BTW, $H \sim 0.85$ at low $t$, in agreement with $\tau_{c}=0.40 \pm 0.05$. A crossover to $H=1 / 2$ is observed for large $t$. The crossover time of course increases with $L$. $H=1 / 2$ corresponds to a process with $C$ exponentially decaying with $t$, or with $C(t, \infty)=\delta_{t, 0}$, as is the case for the M. Thus, the crossover is due to the fact that, beyond the maximal time duration of avalanches, waves are uncorrelated. $H \neq 1 / 2$ implies long range correlations, as we find for the BTW. Furthermore, $H>1 / 2$ corresponds to persistency: an increasing or decreasing trend of $y$ in the past mostly implies a similar tendency in the future. This accounts for the observed expansion and contraction phases in avalanche growth [13]. The above features of $C$ should be responsible for the peculiar long range on-site correlations of the noise expected when mapping the BTW onto a discrete interface growth equation [16].

The BTW has been shown to display a non constant gap in the high $q$ moments of some avalanche PDF's [4. Thus, it should not surprise if, unlike assumed in Eq. (4), also $C$ would manifest similar multifractal properties. Indeed, a more accurate analysis shows that, for high $q$, the gap $d \sigma_{c}(q) / d q$ grows slowly with $q$, beyond the above estimate of $D_{c}$. This multifractal character can be embodied in the more general scaling ansatz [4]

$$
C\left(t=L^{\alpha}, L\right)=L^{f_{c}(\alpha)-\alpha}, \quad(L \gg 1)
$$

with a nonlinear singularity spectrum $f_{c}>-\infty$ in an $\alpha$-interval covering the whole range of possible gaps and linked to $\sigma_{c}$ by Legendre transform. $C$ would satisfy the FFS ansatz (2) only if $f_{c}$ were a linear function, i.e. $f_{c}(\alpha)=-\left(\tau_{c}-1\right) \alpha$ if $\alpha \in\left[0, D_{c}\right], f_{c}(\alpha)=-\infty$ otherwise. The FSS picture given above is in fact only an approximation. This explains also the slight curvature of the $F$ plot for low $t$, which makes the direct measurement of $H$ ambiguous (Fig. 3). Rather than attempting 
a more precise determination of $\sigma_{c}$ and $f_{c}$, below we clarify the difference between BTW and $\mathrm{M}$, and the origin of multiscaling in the former, in the light of probabilistic concepts.

Waves have a relatively simple behavior. So, in a renormalization group (RG) spirit, we can coarse grain the time, by looking at the PDF, $P^{(n)}(s, L)$, of the sum of the sizes of $n$ consecutive waves, regardless of the avalanche they belong to. Since avalanches are constituted by an infinite number [17] of waves for $L \rightarrow \infty$, by sending also $n \rightarrow \infty$, we expect $P^{(n)}$ to approach the PDF of avalanche sizes as a RG fixed point. This approach can be monitored on the basis of the effective moment scaling exponents defined by

$$
\left\langle s^{q}\right\rangle_{n, L}=\int d s s^{q} P^{(n)}(s, L) \sim L^{\sigma_{s, n}(q)} .
$$

For the $\mathrm{M}, \sigma_{s, n}(q)$ does not depend on $n$, and, within our accuracy, is equal from the start to $\sigma_{s}(q)$, such that $\int d s s^{q} P(s, L) \sim L^{\sigma_{s}(q)}$, with $P(s, L)$ representing the avalanche size PDF. To the contrary, in the BTW, as $n$ increases, $\sigma_{s, n}(q)$ varies and moves gradually towards the appropriate $\sigma_{s}(q)$ (Fig. (4).

The result for the $M$ can be explained on the basis of the fact that PDF's of independent variables, satisfying a scaling ansatz of the type (6), have a spectrum which does not change under convolution [18. For example, if we put $P_{w}\left(s=L^{\alpha}, L\right) \sim L^{f_{w}(\alpha)-\alpha}$, where $f_{w}$ is the spectrum of the wave size $\mathrm{PDF}$, one can verify that

$$
\begin{aligned}
& P^{(2)}\left(L^{\alpha}, L\right)= \\
& \int d\left(L^{\beta}\right) P_{w}\left(L^{\alpha}-L^{\beta}, L\right) P_{w}\left(L^{\beta}, L\right) \sim L^{f_{w}(\alpha)-\alpha} .
\end{aligned}
$$

Thus, also the spectrum associated with $P^{(n)}(s, L)$, which is the convolution of $n P_{w}$ 's, does not depend on $n$. This implies the $n$-independence of $\sigma_{s, n}(q)$, which is determined once $f_{w}$ is given. Now, let $P(s, m, L)$ be the probability of having an avalanche with $s$ topplings and $m$ waves: due to the uncorrelated character of different wave sizes, for the $\mathrm{M} P(s, m, L)$ is also the convolution of $m P_{w}$ 's, i.e $P(s, m, L)=P^{(m)}(s, L)$. Therefore, if $P(m, L)$ is the PDF of the total number, $m$, of waves in an avalanche, one has

$$
P(s, L)=\sum_{m} P^{(m)}(s, L) P(m, L),
$$

such that

$$
L^{\sigma_{s}(q)} \sim \sum_{m} L^{\sigma_{s, m}(q)} P(m, L),
$$

which, together with the above results, imply $\sigma_{s, n}(q)=$ $\sigma_{s}(q)$. The coarse graining of waves in the $\mathrm{M}$ does not modify the block PDF spectrum, which is from the start at its fixed point, representing also the scaling of avalanches.
In the BTW case, the nontrivial composition of correlated waves is responsible for the change with $n$ of the effective singularity spectrum of $P^{(n)}$ (Fig. A). For $n=1$, the simple linear FSS form $f_{w}(\alpha)=-\left(\tau_{w}-1\right) \alpha$ $\left(\alpha \in\left[0, D_{w}\right]\right)$ applies, while for $n \rightarrow \infty$ one should recover the nonlinear form needed to describe the complex scaling of $P(s, L)$ [4]. In practice, sampling limitations prevent us from reaching very high $n$. However, even if convergence is relatively slow, the tendency of $\sigma_{s, n}(q)$ to move towards $\sigma_{s}(q)$ is very manifest. We could clearly detect the increase with $n$ of the gap $d \sigma_{s, n}(q) / d q$, at fixed high $q$ : for example, we determined $d \sigma_{s, n}(4) / d q \simeq 2.00,2.20,2.25,2.38$, for $n=1,8,12,24$, respectively. Furthermore, while the asymtoptic gap for $P_{w}(s, L)$ gets readily to the maximum value, i.e. $d \sigma_{s, 1}(q) / d q=2$ for $q \geq 1$, as soon as $n>1$ a constant gap could not be detected for $\sigma_{s, n}(q)$; this confirms the progressive appearance of effective multifractal scaling for the block variable.

In summary, a comparative analysis of wave time series shows that the different forms of universal scaling in the BTW and $\mathrm{M}$ are determined by distinct dynamical correlation patterns. For the $\mathrm{M}$ case, the wave level of description is in fact coarse grained enough to fully account, without further modifications, also for the avalanche level. Waves are uncorrelated and their PDF, satisfying FSS, coincides with the avalanche PDF, as far as exponents are concerned. To the contrary, in the BTW, under coarse graining, long time correlations substantially modify the scaling properties of waves, determining also multiscaling features. We regard these as prototype mechanisms to be generally expected in sandpile and similar SOC models. The 2D BTW behavior is probably less generic than the $\mathrm{M}$ one. For example, in the 3D BTW, the fact that avalanches are constituted by an average number of waves which remains finite for $L \rightarrow \infty$, strongly suggests a M type mechanism and FSS for the avalanche PDF. Indeed, even if subsequent waves are not strictly uncorrelated, like in the 2D Manna model, $C$ should decay extremely fast, with an $L$ independent time cutoff. Thus, coarse graining in time can not substantially modify the PDF of block variables, with respect to $P_{w}$. We expect the exactly known wave exponents to apply also to the avalanche size PDF. Indeed, numerically determined avalanche exponents for 3D BTW turn out to be strikingly close to the wave values 10 .

We thank C. Tebaldi and C. Vanderzande for useful discussions. Partial support from the European Network Contract No. ERBFMRXCT980183 is acknowledged.

[1] P. Bak, C. Tang, and K. Wiesenfeld, Phys. Rev. Lett. 59, 381 (1987); Phys. Rev. A 38, 364 (1988).

[2] T. Hwa and M. Kardar, Phys. Rev. B 45, 7002 (1992). 
[3] P. Grassberger and S. S. Manna, J. Phys. (France) 51, 1077 (1990); S. S. Manna, Physica A 179, 249 (1991); S. Lübeck and K. Usadel, Phys. Rev. E 55, 4095 (1997).

[4] C. Tebaldi, M. De Menech, and A. L. Stella, Phys. Rev. Lett. 83, 3952 (1999).

[5] S. S. Manna, J. Phys. A 24, L363 (1991).

[6] A. Chessa, A. Vespignani, and S. Zapperi, Compu. Phys. Comm. 121-122, 299 (1999).

[7] S. Lübeck, Phys. Rev. E 61, 204 (2000).

[8] D. Dhar, Physica A 263, 4 (1999).

[9] E. V. Ivashkevich, D. V. Ktitarev, and V. B. Priezzhev, Physica A 209, 347 (1994).

[10] D. V. Ktitarev, S. Lübeck, P. Grassberger, and V. B. Priezzhev, Phys. Rev. E 61, 81 (2000).

[11] V. B. Priezzhev., E. V. Ivashkevich, and D. V. Ktitarev, Phys. Rev. Lett. 76, 2093 (1996).

[12] M. Paczuzki and S. Boettcher, Phys. Rev. E 56, R3745 (1997).

[13] D. V. Ktitarev and V. B. Priezzhev, Phys. Rev. E 58, 2883 (1998).

[14] M. De Menech, A. L. Stella, and C. Tebaldi, Phys. Rev. E 58, R2677 (1998).

[15] R. V. Sole, S. C Manrubia, M. Benton, and P. Bak, Nature 388, 764 (1997).

[16] M. J. Alava and K. B. Lauritsen, to be published.

[17] Its average grows $\sim \log L$ for both BTW and M.

[18] This holds irrespective of the linear or nonlinear nature of the spectrum of the independent variables.

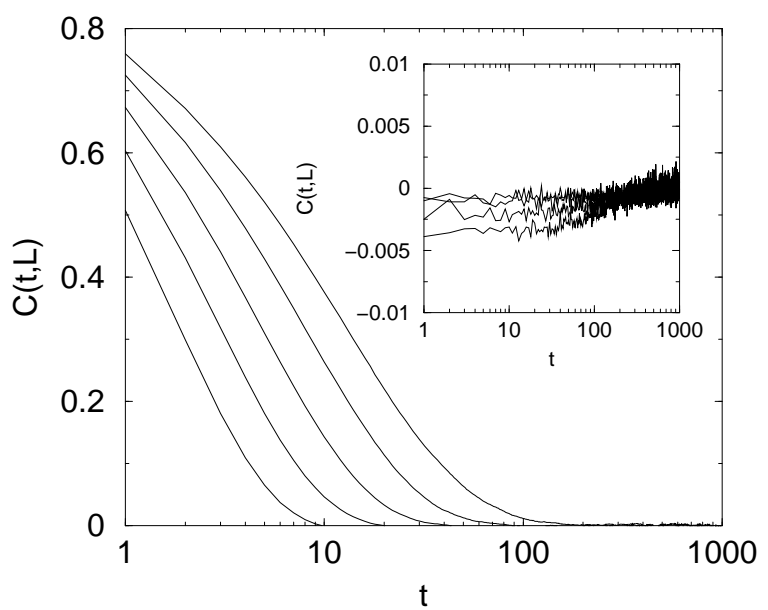

FIG. 1. For the BTW model, as $L$ grows, $C$ decays more and more slowly, ( $L=128,256,512,1024,2048$ from left to right). $C(t \geq 1, L) \simeq 0$ for the $\mathrm{M}$ (inset).

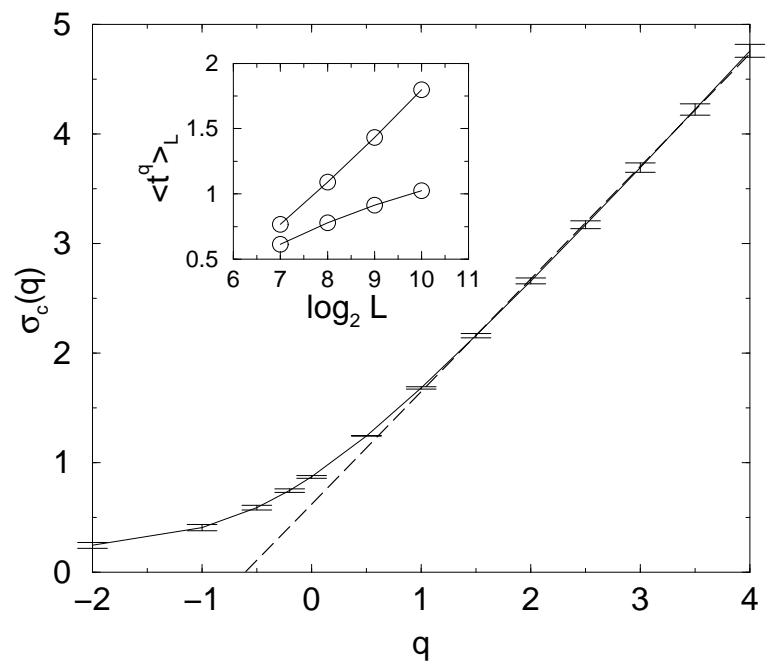

FIG. 2. The dashed curve interpolates $\sigma_{c}(q)$ for $1 \leq q \leq 4$; it has slope $D=1.02$ and intercept 0.62 . The inset shows semi-logarithmic plots of $\left\langle t^{q}\right\rangle_{L}$ vs $\log _{2} L$ for $q=-1,-0.5$.

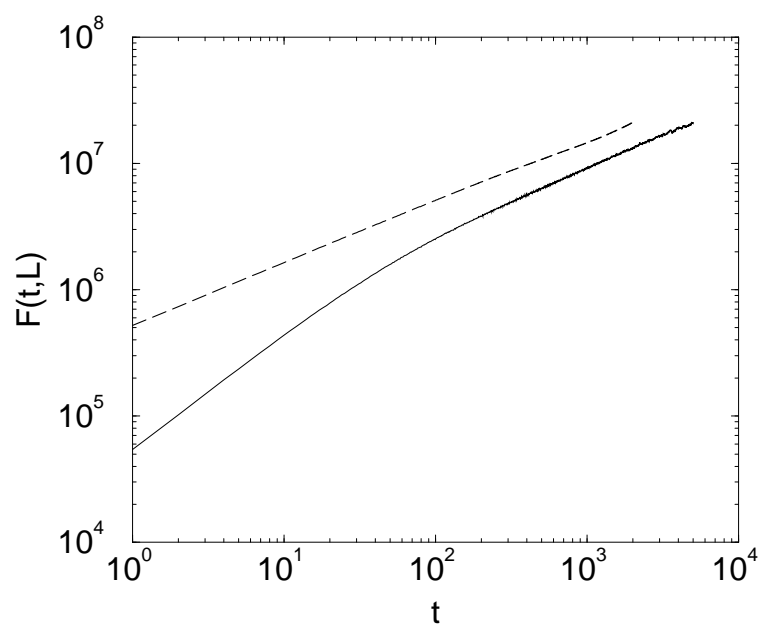

FIG. 3. Plots of $F(t, L=2048)$ for the BTW (continuos) and the M (dashed, slope $=0.5)$. 


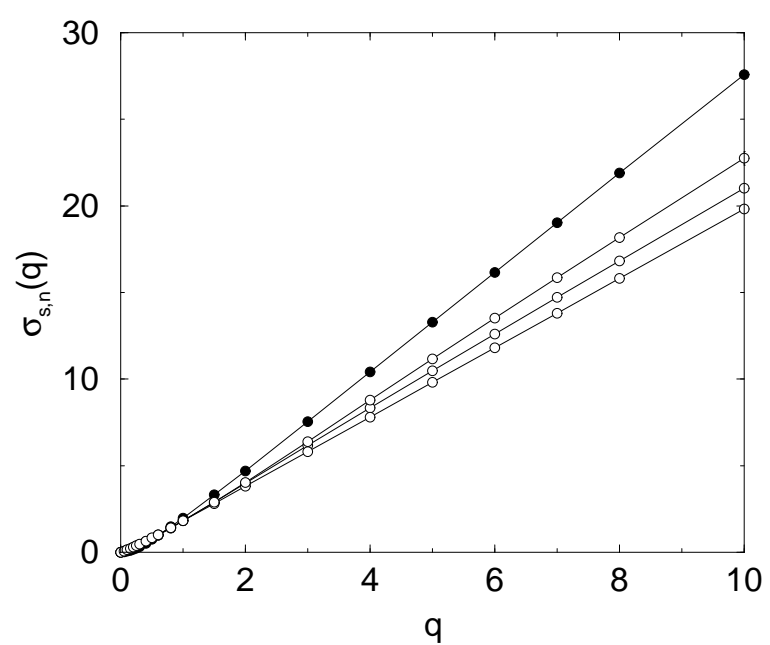

FIG. 4. $\sigma_{s, n}(q)$ for the BTW, $n=1,8,24$ (open circles, from the bottom). The full circles show $\sigma_{s}(q)$. 